koppelungsschleife ist in der repräsentativen Demokratie substantielle Repräsentation über die Kontrolle der zeitlich limitierten Macht durch die Wählerschaft realisierbar. ${ }^{27}$ Dies wird in den nordrhein-westfälischen Großstädten durch den Fall der Fünfprozenthürde aber erheblich schwerer. Nicht nur, dass unklare Mehrheitsverhältnisse und damit unklare Verantwortlichkeiten forciert wurden, sondern die hierdurch eingezogenen kleinen Gruppierungen können kaum effektiv das Votum ihrer Wählerschaft in die Kommunalparlamente einbringen. Erschwerend kommt hinzu, dass diese sich nicht selten zu immer wieder neuen Fraktionen zusammenschließen, um an den Zuwendungen partizipieren zu können, während die gemeinsame inhaltliche Basis dieser neuen Fraktionen oft unklar bleibt. Daher ist es der Wählerschaft selbst bei starkem politischen Interesse kaum möglich, die Arbeit der ursprünglich gewählten Gruppierungen zu beurteilen und damit zu kontrollieren.

Eine einheitliche gesetzliche Sperrklausel von 2,5 Prozent im nordrhein-westfälischen Kommunalwahlrecht könnte die Fragmentierung und Zersplitterung der Großstadtparlamente maßgeblich reduzieren, ohne dass in den unteren bis mittleren Gemeindegrößenklassen der Grundsatz der Wahlgleichheit signifikant eingeschränkt würde. In diesen Gemeinden hat die Aufhebung gesetzlicher Sperrklauseln zu weniger Problemen in den Kommunalparlamenten geführt, auch weil hier im stärkeren Maße die natürliche Hürde wirkt. Eine einheitliche Sperrklausel von 2,5 Prozent liegt kaum über dieser natürlichen in kleineren und mittleren Kommunen und würde damit den hier deutlich geringeren Problemen der Fragmentierung angemessen Rechnung tragen. ${ }^{28}$

27 Vgl. Sieglinde Rosenberge / Gilg Seeber, Wählen, Wien 2008.

28 Vgl. Friedrich Pukelsheim / Sebastian Maier / Peter Leutgäb, a.a.O. (Fn. 24).

\title{
Der geliebte Verräter - Zum Einfluss von Spitzenkandidaten auf das Wahlverhalten am Beispiel der saarländischen Landtagswahl 2009
}

\author{
Johannes N. Blumenberg und Manuela S. Kulick
}

Wahlen - und Bundestagswahlen im Besonderen - sind das Ereignis, bei dem die Mehrzahl der Bürger ihre Einstellungen und Meinungen in tatsächliches politisches Verhalten umsetzt. Zugleich ist dies für die meisten auch die einzige aktive Teilnahme an der Politik. Es ist also nicht übertrieben, den Wahlakt als wichtigstes demokratisches Element (nicht nur) im deutschen politischen System zu bezeichnen.

War das Wahlverhalten lange Zeit von Gruppenzugehörigkeit determiniert, so hat sich in den letzten Jahren in der Wahlforschung die Annahme verfestigt, dass immer mehr situative Komponenten, persönliche Vorlieben und Sympathien, beispielsweise die Kandidatenorientierung, bei der tatsächlichen Wahlentscheidung eine große Rolle spielen. Dazu soll hier das kleinste Flächenbundesland, das Saarland, betrachtet werden, wo im Vorfeld der letzten Landtagswahl eine stark auf die Rolle des Kandidaten Oskar Lafontaine fokussierte Medienberichterstattung stattfand, bei der immer wieder auf die tragende Rolle des 
ehemaligen SPD-Vorsitzenden, ehemaligen Ministerpräsidenten und Zugpferdes der Partei Die Linke im westlichen Teil der Bundesrepublik rekurriert wurde. ${ }^{1}$ Vor diesem Hintergrund stellt sich auch aus Sicht der Wissenschaft die Frage, ob der Kandidat wirklich die Rolle, die ihm die Medien attestierten, bei den Wählern der Linken eingenommen hat.

Dass der Kandidat einer Partei wesentlichen Einfluss auf das Verhalten der Wähler haben kann, konnten schon Gabriel und Neller zeigen, die den Einfluss der Spitzenkandidaten für die Bundestagswahlen 1994, 1998 und 2002 untersuchten und zu dem Ergebnis kamen, dass die Kandidatenpräferenz bei allen drei Wahlen wichtig für die Wahlentscheidung war und in den meisten Fällen sogar die Themenorientierung übertraf. ${ }^{2}$ Brettschneider stellte darüber hinaus fest, dass es dabei zu keiner Entpolitisierung der Kandidatenwahrnehmung kommen muss. Die Wahlentscheidung wird zwar auch aufgrund der Kandidaten getroffen, diese werden jedoch mit politischen Standpunkten und ihrer Themenkompetenz verbunden, so dass die Wahlentscheidung aufgrund eines Kandidaten nicht zwangsläufig von der Themenorientierung losgelöst ist. ${ }^{3}$

\section{Der Fall Saarland}

Am 30. August 2009 wurde im Saarland - kurz vor der Bundestagswahl 2009 - ein neuer Landtag gewählt. Die Wahlbeteiligung war im Vergleich zur Wahl 2004 um zwölf Prozentpunkte angestiegen, nachdem sie bei den beiden vorherigen Wahlen drastisch gesunken war (vgl. Abbildung 1). Entgegen dem allgemeinen Trend bei Landtagswahlen, nach dem die Wahlbeteiligung tendenziell eher niedrig ist, hatte das Saarland mit dieser Problematik bis Ende der 1990er Jahre nicht zu kämpfen. Vor 1999, als die Wahlbeteiligung drastisch fiel ${ }^{4}$, lag diese im Saarland stets über 80 Prozent.

1 Vgl. Mechthild Küpper, Oskars Schicksalswahl, in: FAZ vom 15. August 2009, S. 8.

2 Vgl. Oscar W. Gabriel/ Katja Neller, Kandidatenorientierungen und Wahlverhalten bei den Bundestagswahlen 1994-2002, in: Jürgen W. Falter / Oscar W. Gabriel / Bernhard Weßels (Hrsg.), Wahlen und Wähler - Analysen aus Anlass der Bundestagswahl 2002, Wiesbaden 2005, S. 213 - 243.

3 Vgl. Frank Brettschneider, Spitzenkandidaten und Wahlerfolg. Personalisierung - Kompetenz Parteien, Wiesbaden 2002.

4 Die Wahlbeteiligung sank um knapp 15 Prozent, was zwischen zwei Wahlen ungewöhnlich viel ist. Zwar sinkt die Wahlbeteiligung auf allen politischen Ebenen (auf unterschiedlichen Niveaus), allerdings handelt es sich für gewöhnlich um einen eher langsamen Prozess. Wird bei der Landtagswahl 1999 neben der prozentualen Verteilung der Stimmen auch die absolute Verteilung betrachtet, zeigt sich, dass die geringe Wahlbeteiligung hauptsächlich zu Lasten der SPD geht, die einen Großteil ihrer Wähler nicht mobilisieren konnte. Die SPD verlor bei der Landtagswahl 1999 im Vergleich zu der vorherigen Wahl prozentual „nur“ fünf Prozent, in absoluten Wählerstimmen ist der Unterschied erheblich größer. 1994 konnten 340.091 Wähler mobilisiert werden, 1999 nur noch 247.311. Das macht einen Verlust von knapp 30 Prozent aus. Die CDU konnte zwar prozentual Stimmen hinzugewinnen, verlor faktisch aber auch Wähler, wobei der Anteil mit knapp fünf Prozent erheblich kleiner war. Vgl. Jürgen R. Winkler, Die saarländische Landtagswahl vom 16. Oktober 1994: Bestätigung der SPD-Mehrheit, Debakel der FDP, in: ZParl, 26. Jg. (1995), H. 2, S. 249 - 261; ders., Die saarländische Landtagswahl vom 5. September 1999. Die CDU erhält die Macht zurück, in: ZParl, 31. Jg. (2000), H. 1, S. 28 - 42; ders., Die saarländische Landtagswahl vom 5. September 2004. Vom Zwei- zum Vierparteiensystem mit einer dominanten CDU, in: ZParl, 36. Jg. (2005), H. 1, S. 19 - 35; ders., Die saarländische Landtagswahl vom 30. August 2009: Auf dem Weg nach Jamaika, in: ZParl, 41. Jg. (2010), H. 2, S. $339-355$. 


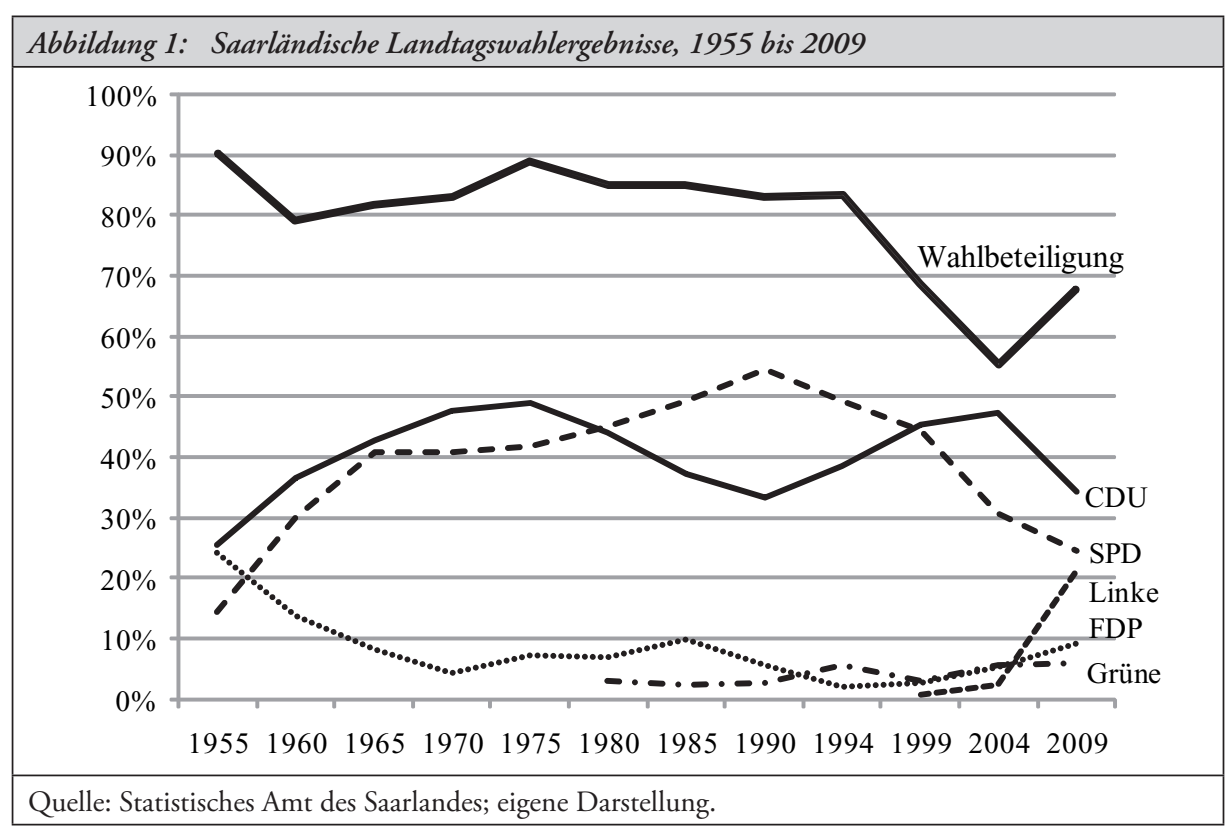

Die geringe Wahlbeteiligung 1999 ging zudem mit einem Regierungswechsel einher. Bis 1998 stellte die SPD mit Oskar Lafontaine den Ministerpräsidenten, der nach 14 Jahren im Amt nach Bonn wechselte und dort im Kabinett Gerhard Schröders den Posten des Finanzministers übernahm. Die SPD unter Ministerpräsident Reinhard Klimmt wurde von den Saarländern nicht wiedergewählt. Stattdessen gaben diese mit nur 1,1 Prozentpunkten Vorsprung der CDU einen Regierungsauftrag. Bei der Landtagswahl 1994 hatten die beiden Parteien noch rund zehn Prozentpunkte auseinandergelegen. 2004 konnte die CDU ihr Ergebnis leicht verbessern, die SPD dagegen verlor weiter und erreichte nur noch 30,8 Prozent der Wähler. Auch bei der Landtagswahl 2009 setzte sich der Abwärtstrend fort (24,5 Prozent). Allerdings war diesmal auch die CDU davon betroffen, die dreizehn Prozentpunkte verlor und auf 34,5 Prozent kam.

Die Linke trat im Saarland erstmals bei der Landtagswahl 1999, damals noch als PDS, in Erscheinung, konnte jedoch nur 0,8 Prozent der Wähler für sich mobilisieren. Fünf Jahre später erhielt die „ostdeutsche Regionalpartei“ bereits 2,3 Prozent, und ein Jahr darauf - bei der Bundestagswahl 2005 - gaben 18,5 Prozent der Saarländer der Linken ihre Stimme. ${ }^{5}$ Auch wenn Die Linke bei der Bundestagswahl 2005 in einigen westdeutschen Bundesländern gut abschnitt, erreichte sie doch nur im Saarland Stimmenanteile im zweistelligen Prozentbereich. Bei der Landtagswahl 2009 setzte die Partei ihren Erfolgskurs fort und konnte 21,3 Prozent der Saarländer für sich gewinnen. Ebenfalls beachtenswert ist die

5 Anzumerken ist hierbei, dass zur Landtagswahl 2004 die PDS antrat, während zu der Bundestagswahl 2005 das Wahlbündnis aus PDS und WASG auf den Stimmzetteln erschien, was das Unbehagen einiger Westdeutscher, ihre Stimme der Linken zu geben, verringert haben dürfte; vgl. zur Entstehung der Linken Sören Messinger / Jonas Rugenstein, Der Erfolg der Partei die Linke - Sammlung im programmatischen Nebel, in: Felix Butzlaff / Stine Harm / Franz Walter (Hrsg.), Patt oder Gezeitenwechsel - Deutschland 2009, Wiesbaden 2009, S. 67 - 93. 
Wahlbeteiligung: Diese stieg erstmals wieder an, auf 67,6 Prozent (gut zwölf Prozentpunkte), was zu einem nicht unwesentlichen Anteil der Linken zuzuschreiben ist, denn sie konnte von ihren „neuen“ Wählern' 42,5 Prozent aus dem Nichtwählerlager rekrutieren. Ehemalige SPD-Wähler machten mit 24,5 Prozent die zweitstärkste Gruppe aus. Aber auch die CDU verlor Wähler an Die Linke. Diese Gruppe stellt mit 9,4 Prozent jedoch nur einen vergleichsweise geringen Anteil der hinzugewonnenen Wähler der Linken. ${ }^{7}$

Bei der Landtagswahl 2009 darf ein weiterer Faktor nicht außer Acht gelassen werden: die zeitliche Nähe zur Bundestagswahl. Dass Landtagswahlen von der „übergeordneten Konstellation im Bundestag "8 beeinflusst werden können, ist mehrfach belegt worden. ${ }^{9} \mathrm{Da}$ die Landtagswahl nur einen Monat vor der Bundestagswahl stattfand, fiel die Zeit des Wahlkampfes - zumindest teilweise - zusammen, so dass eine Verknüpfung zwischen bundes- und landespolitischen Themen und Politikern als gegeben angesehen werden kann.

Um den Erfolg der Linken im Saarland zu erklären, gibt es zwei Möglichkeiten. Zum einen wäre zu vermuten, dass „die Saarländer“ schon immer etwas „linker“ waren und nun, da sich ihnen erstmals die Möglichkeit bot, eine Partei links von der SPD zu wählen, diese Chance ergriffen. Zwar wurde das Land einige Zeit von der SPD regiert, aber es ist nicht sehr wahrscheinlich, dass seine Bürger „linker“ sind oder gänzlich andere politische Präferenzen aufweisen als die Einwohner der anderen westdeutschen Bundesländer. ${ }^{10}$ Viel wahrscheinlicher ist - und dies ist die zweite Möglichkeit -, dass ein anderer Faktor für das gute Abschneiden der Linken im Saarland, sowohl bei der Bundestagswahl 2005 als auch bei der Landtagswahl 2009, verantwortlich ist. Dieser Faktor heißt Oskar Lafontaine. Er ist für die Saarländer nicht einfach „nur“ ein ehemaliger SPD-Spitzenpolitiker, der sich entschlossen hat, das Wahlbündnis zwischen PDS und WASG und schließlich den Zusammenschluss der beiden Parteien zur Linken mit zu gestalten. Lafontaine war lange Zeit Ministerpräsident im Saarland und erreichte dabei absolute Mehrheiten für seine Partei, mit der Folge, dass er das Land ohne Koalitionspartner regieren konnte. Die Vermutung liegt nahe, dass die Linke im Saarland insbesondere viele ehemalige SPD-Anhänger für sich gewann, denn diese verbinden mit der Linken verstärkt auch Oskar Lafontaine, den sie bereits als langjährigen Ministerpräsidenten schätzen lernten. ${ }^{11}$ Dass SPD-Anhänger zur Linken gewechselt

6 Im Vergleich zu der Landtagswahl 2004 bestand die Wählerschaft der Linken 2009 zu 89,2 Prozent aus neuen Wählern.

7 Siehe dazu die Wählerwanderungsbilanz von Infratest dimap, Wahlberichterstattung im Auftrag der ARD.

8 Rainer Dinkel, Der Zusammenhang zwischen Bundes- und Landtagswahlergebnissen, in: PVS, 18. Jg. (1977), H. 1, S. 348 - 359, S. 357.

9 Vgl. ebenda; Simone Burkhart, Parteipolitikverflechtung. Über den Einfluss der Bundespolitik auf Landtagswahlentscheidungen von 1976 bis 2000, in: PVS, 46. Jg. (2005), H. 1, S. 14 - 38, sowie der Forschergruppe um Oscar W. Gabriel und Everhard Holtmann, siehe dazu die Beiträge in Heft 3/2007 der ZParl.

10 Bei einem Vergleich der Links-Rechts-Selbsteinstufung der Saarländer mit der Grundgesamtheit im Datensatz, ergibt sich eine durchschnittliche Differenz von lediglich 0,04 Punkten auf einer 11 er Skala. Auch die Verteilung der beiden Gruppen verläuft nach einem ähnlichen Muster, so dass die Daten die Aussage bestätigen, dass bei den Saarländern keine linkeren Tendenzen vorzufinden sind.

11 Vgl. Jürgen W. Winkler, Das Parteiensystem des Saarlandes, in: Uwe Jun / Melanie Haas / Oskar Niedermayer (Hrsg.), Parteien und Parteiensysteme in den deutschen Ländern, Wiesbaden 2008, S. $369-386$, S. $378 \mathrm{ff}$. 
sind, lässt nicht nur das Wahlergebnis vermuten, sondern wird auch durch die Wählerwanderungsbilanz von Infratest dimap bestätigt. ${ }^{12}$

Neben der Rekrutierung aus den Reihen der SPD konnte Die Linke auch verstärkt Nichtwähler für sich gewinnen, die wiederum teilweise frühere SPD-Wähler gewesen sein mögen. Seit dem Regierungswechsel 1999 hat die SPD 20 Prozent ihrer Wähler verloren, und zwar hauptsächlich an das Nichtwählerlager, was sich auch an der gesunkenen Wahlbeteiligung zeigt. Mit der Landtagswahl 2009 stieg die Wahlbeteiligung an - aber nicht die SPD profitierte davon, sondern vielmehr ihr schärfster Konkurrent: Die Linke.

Ist das gute Abschneiden der Linken nun ausschließlich auf Oskar Lafontaine zurückzuführen, oder sind die Saarländer doch ein wenig linker als die restlichen westdeutschen Bundesbürger? Um dies zu entscheiden, wird im Folgenden nicht nur der Spitzenkandidat der Linken - die als einzige Partei neben den beiden Volksparteien einen Kandidaten für das Amt des Ministerpräsidenten kürte - betrachtet, sondern es werden auch andere Faktoren untersucht, die die Wahlentscheidung beeinflussen können. Dazu zählen, neben dem Kandidaten, bekanntermaßen die Parteiidentifikation sowie Sachthemen.

\section{Datenlage und Operationalisierung}

Für die Analyse wurde auf Daten des Multi-Level-Panels (MLP) zurückgegriffen, die im Rahmen der German Longitudinal Election Study erhoben wurden. ${ }^{13}$ Beim MLP handelt es sich um eine dreiwellige Online-Panelbefragung, die in den fünf Bundesländern, in denen im Vorfeld der Bundestagswahl eine Landtagswahl stattfand, durchgeführt wurde. Dabei wurden nur Fälle aus der ersten Panelwelle für das Saarland, Sachsen, Brandenburg und Thüringen berücksichtigt. ${ }^{14}$

Zur Berechnung wird auf die sozialpsychologische Schule der Wahlforschung, den AnnArbor-Ansatz, zurückgegriffen. Dieser versucht das Wahlverhalten nicht auf objektive Faktoren wie Einkommen oder Zugehörigkeit zu einer sozialen Gruppe zurückzuführen, sondern nimmt explizit auf persönliche Einstellungen Bezug. ${ }^{15}$ Eine Schlüsselrolle spielt dabei

12 Siehe dazu die Wählerwanderungsbilanz von Infratest dimap, Wahlberichterstattung im Auftrag der ARD.

13 Die Daten, die diesem Beitrag zugrunde liegen, wurden im Rahmen der German Longitudinal Election Study (Komponente Y: Multi-Level-Panel) erhoben. Die Erhebung geschah im Auftrag von Hans Rattinger (Universität Mannheim), Sigrid Roßteutscher (Universität Frankfurt), Rüdiger Schmitt-Beck (Universität Mannheim) und Bernhard Weßels (Wissenschaftszentrum Berlin für Sozialforschung) in enger Zusammenarbeit mit der DGfW und GESIS - Leibniz-Institut für Sozialwissenschaften. GESIS ist auch für die Datenaufbereitung und -dokumentation verantwortlich und stellt die Daten für Analysen zur Verfügung. Weder die genannten Personen noch die beteiligten Institute tragen Verantwortung für die Analyse oder Interpretation der Daten in diesem Beitrag.

14 Aufgrund von extrem hoher Panelmortalität war es nicht möglich, auch die anderen Wellen in die Analyse mit aufzunehmen, da die Fallzahlen, insbesondere für das Saarland, zu klein wurden. Auch konnte Schleswig-Holstein - wo am Tag der Bundestagswahl ein neuer Landtag gewählt wurde nicht in die Analyse mit aufgenommen werden. Zum Start der ersten Welle des MLP war noch nicht bekannt, dass auch in Schleswig-Holstein 2009 eine Landtagswahl stattfinden würde.

15 Zur genaueren Beschreibung des Ansatzes siehe beispielsweise Harald Schoen / Cornelia Weins, Der sozialpsychologische Ansatz zur Erklärung von Wahlverhalten, in: Jürgen W. Falter / Harald Schoen (Hrsg.), Handbuch Wahlforschung, Wiesbaden 2005, S. 187 - 242, S. 188 ff. 
die Parteiidentifikation, die über die Selbsteinstufung der Befragten gemessen wird. Weiterhin steht die Parteiidentifikation in wechselseitiger Beziehung zu den beiden anderen Faktoren des Modells: der Kandidaten- und der Themenorientierung (Issues).

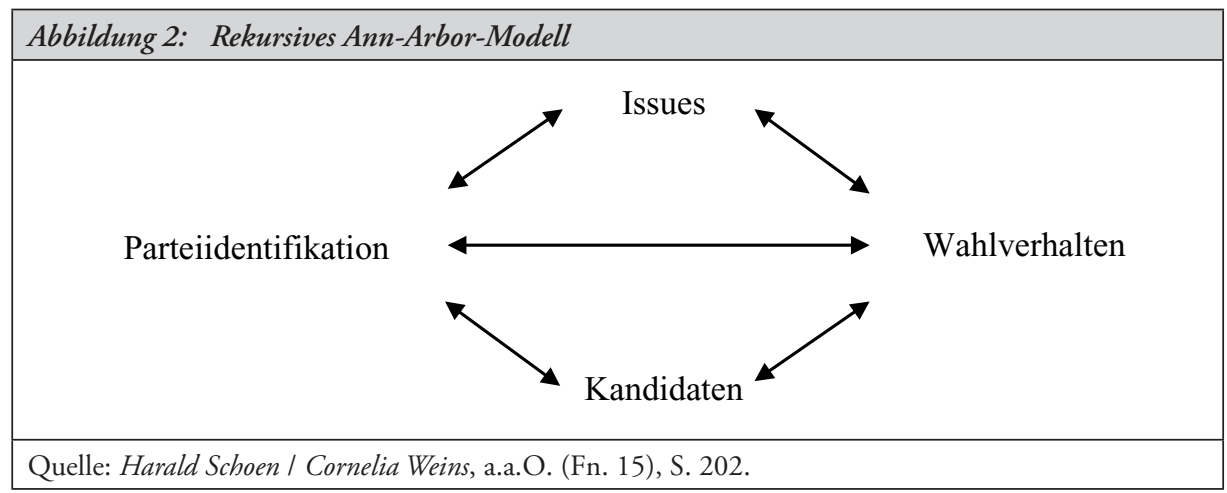

Entgegen der weit verbreiteten Vorgehensweise, mit dem Modell der multinominalen logistischen Regression zu arbeiten, wird hier dem Verfahren der binär logistischen Regression der Vorzug gegeben, um das Wahlverhalten zu analysieren. So weisen Fürnberg / Schnapp ${ }^{16}$ wie auch Völkl ${ }^{17}$ in Bezug auf Thurner ${ }^{18}$ darauf hin, dass die Anwendungsvoraussetzung der multinominalen logistischen Regression verletzt werden könnte, wenn als abhängige Variable das Wahlverhalten verwendet wird, da die einzelnen Kategorien nicht unbedingt voneinander unabhängig betrachtet werden können. ${ }^{19}$ Die Unabhängigkeit von irrelevanten Alternativen ist jedoch eine zwingende Voraussetzung der multinominalen Regression. Es ist anzunehmen, dass insbesondere durch das Antreten der Linken die Auswahlwahrscheinlichkeit der Wähler zwischen SPD und CDU von koalitionsstrategischen Überlegungen beeinflusst wurde. ${ }^{20}$

Entsprechend der Determinanten-Trias des Ann-Arbor Ansatzes finden die Parteiidentifikation sowie die Kandidatenorientierung ${ }^{21}$ und die Themen als unabhängige Variablen Eingang in das logistische Regressionsmodell. Die Parteiidentifikation gilt dabei als der

16 Vgl. Ossip Fürnberg / Kai-Uwe Schnapp, Magdeburger Modell und „rote Laterne“: Wahlen in Sachsen-Anhalt, in: Kerstin Völkl / Kai-Uwe Schnapp / Everhard Holtmann / Oscar W. Gabriel (Hrsg.), Wähler und Landtagswahlen in der Bundesrepublik Deutschland, Baden-Baden 2008, S. $381-410$, S. 398.

17 Vgl. Kerstin Völkl, Reine Landtagswahlen oder regionale Bundestagswahlen? Eine Untersuchung des Abstimmungsverhaltens bei Landtagswahlen 1990-2006, Baden-Baden 2009, S. 211.

18 Vgl. Paul W. Thurner, Modelle diskreter Entscheidung, in: Joachim Behnke / Thomas Gschwend I Delia Schindler / Kai-Uwe Schnapp (Hrsg.), Methoden der Politikwissenschaft, Baden-Baden 2006, S. 193 - 204, S. 195.

19 Auch Dieter Fuchs und Steffen Kühnel gehen bereits in ihrem Aufsatz auf das Problem der Unabhängigkeit von irrelevanten Alternativen ein. Die Überlegungen Thurners sind insofern nicht neu; vgl. dies., Wählen als rationales Handeln. Anmerkungen zum Nutzen des Rational-Choice-Ansatzes in der empirischen Wahlforschung, in: Hans-Dieter Klingemann / Max Kaase (Hrsg.), Wahlen und Wähler. Analysen aus Anlaß der Bundestagswahl 1990, Opladen 1994, S. 305 - 364, S. 330.

20 Vgl. Kerstin Völkl, a.a.O. (Fn. 17).

21 Die Kandidatenorientierung wird mit Hilfe der Politiker-Skalometer gemessen. Diese wurden für alle Landesvorsitzenden der größeren Parteien abgefragt. 
stabilste der drei Faktoren, festigt sich durch die wiederholte Wahl einer bestimmten Partei und ändert sich - einmal erworben - nur in Ausnahmefällen. ${ }^{22}$

Die Wahlentscheidung wird als abhängige Variable behandelt und nach dem Muster „(1) Partei genannt“ und „(0) Partei nicht genannt“ für die einzelnen Parteien gebildet. Parteiidentifikation und Kandidatenorientierung bleiben als Skalen vorhanden und werden auf einen Wertebereich von 0 (keine Parteiidentifikation mit Partei X) bis 1 (sehr starke Parteiidentifikation mit Partei X) beziehungsweise 0 (halte überhaupt nichts von dieser Person) bis 1 (halte sehr viel von dieser Person) normiert. Die Themen-Variable wird aus der vermuteten Lösungskompetenz zum wichtigsten und zweitwichtigsten Problem gebildet. Hierbei zählt keine Nennung als „0“, die Nennung der Partei beim zweitwichtigsten, aber nicht beim wichtigsten Problem als „0,33“, die Nennung beim wichtigsten, aber nicht beim zweitwichtigsten Problem als „0,66“ und die Nennung der jeweiligen Partei bei den beiden wichtigsten Problemen als „1“. Diese Vorgehensweise hat sich bereits bei Kellermann ${ }^{23}$ bewährt.

\section{Wahlentscheidende Faktoren}

Für das Saarland wurden entsprechend fünf binominale logistische Regressionsmodelle berechnet. Die Kandidaten aller Parteien in das Modell aufzunehmen ist dabei sinnvoll, da Kandidaten sowohl positiven als auch negativen Einfluss auf die Wahlentscheidung ausüben können. Es ist beispielsweise möglich, dass ein tendenziell der SPD zugeneigter Wähler der Partei am Wahltag seine Stimme verweigert, da er den Kandidaten nicht akzeptieren kann.

Tabelle 1: Einfluss der Faktoren auf die Wablentscheidung im Saarland

\begin{tabular}{|l|c|c|c|r|r|r|r|r|}
\hline & $\begin{array}{c}\text { Nagelkerkes } \\
\mathrm{R}^{2}\end{array}$ & PI $^{*}$ & Issue $^{*}$ & $\begin{array}{c}\text { Kandidat } \\
\text { CDU }\end{array}$ & $\begin{array}{c}\text { Kandidat } \\
\text { SPD }\end{array}$ & $\begin{array}{c}\text { Kandidat } \\
\text { FDP }\end{array}$ & $\begin{array}{c}\text { Kandidat } \\
\text { Grüne }\end{array}$ & $\begin{array}{c}\text { Kandidat } \\
\text { Linke }\end{array}$ \\
\hline CDU & 0,811 & $79,948^{\mathrm{a}}$ & $5,504^{\mathrm{c}}$ & $15817,885^{\mathrm{a}}$ & 8,826 & 0,359 & 0,369 & $0,107^{\mathrm{c}}$ \\
\hline SPD & 0,624 & $47,063^{\mathrm{a}}$ & 1,631 & $0,074^{\mathrm{b}}$ & $614,446^{\mathrm{a}}$ & 0,382 & 0,370 & $0,199^{\mathrm{c}}$ \\
\hline FPD & 0,593 & $386,762^{\mathrm{a}}$ & 0,969 & 1,094 & $0,009^{\mathrm{b}}$ & $65,279^{\mathrm{c}}$ & 2,227 & 0,410 \\
\hline Grüne & 0,534 & $631,609^{\mathrm{a}}$ & 0,316 & 0,805 & 0,072 & 3,604 & 12,432 & 0,240 \\
\hline Linke & 0,671 & 2,518 & $14,355^{\mathrm{b}}$ & 0,189 & 0,149 & 2,382 & 0,337 & $230,070^{\mathrm{a}}$ \\
\hline
\end{tabular}

* Parteiidentifikation / Issueorientierung zur gewählten Partei.

Signifikanzen: a: $\mathrm{p}<0,000$; $\mathrm{b}: \mathrm{p}<0,01 ; \mathrm{c}: \mathrm{p}<0,05$.

Quelle: Eigene Berechnung auf Basis des MLP.

Die Erklärungskraft des Modells ist für alle Parteien als gut zu bezeichnen (siehe Tabelle 1). Mit einem Nagelkerkes $\mathrm{R}^{2}$ von 0,811 wird die Varianz der unabhängigen Variablen für die CDU am besten erklärt. Aber auch die Wahlentscheidung für Die Linke, mit einem Erklärungsanteil von 67,1 Prozent, sowie für die SPD, mit 62,4 Prozent, können gut von den

22 Vgl. dazu ausführlich Stefan Dablem, Wahlentscheidung in der Mediengesellschaft, Freiburg 2001, S. 77 ff.; Wilhelm Bürklin / Markus Klein, Wahlen und Wählerverhalten, Opladen 1998, S. 66 f.

23 Vgl. Charlotte Kellermann, „Trends and Constellations”: Bestimmungsfaktoren des Wahlverhaltens bei den Bundestagswahlen 1990-2005, in: Hans Rattinger / Oscar W. Gabriel / Jürgen W. Falter (Hrsg.), Der gesamtdeutsche Wähler, Baden-Baden 2002, S. 297 - 327. 
ausgewählten Variablen erklärt werden. Dabei unterscheiden sich jedoch die Einflussstärken der erklärenden Variablen teilweise beträchtlich.

Einen hohen signifikanten Einfluss hat bei der Wahlentscheidung für die CDU, SPD, FDP und die Grünen die Parteiidentifikation. Allerdings wird dieser Faktor bei CDU und SPD noch von dem Einfluss des jeweiligen Kandidaten übertroffen. Dies ist nur bedingt erstaunlich, wenn man bedenkt, dass die beiden Spitzenkandidaten, Ministerpräsident Peter Müller und sein Herausforderer Heiko Maas, direkt um das Amt des Ministerpräsidenten konkurrierten. Weil beide bereits lange in der Landespolitik aktiv sind und damit von den Wählern als Repräsentanten ihrer Parteien wahrgenommen werden, sind sie vielen Bürgern ein Begriff und direkte Bezugsgröße bei der Wahlentscheidung. Bei den kleineren Parteien zeigen sich Unterschiede in der Höhe und Signifikanz der unabhängigen Variablen. Nagelkerkes $\mathrm{R}^{2}$ weist für die FDP und die Grünen einen deutlich geringeren Erklärungsanteil auf als für Die Linke. Wie bei den großen Parteien dominiert bei beiden der Einfluss der Parteiidentifikation (die Wahlentscheidung der Grünen lässt sich ausschließlich darüber erklären), und der eigene Kandidat hat nur bei der FDP (Christoph Hartmann) einen signifikanten, aber vergleichsweise geringen Einfluss.

\subsection{Parteiidentifikation}

Die Faktoren der Linken zeichnen ein anderes Bild. Das Modell zeigt mit einem Erklärungsanteil von 67,1 Prozent einen vergleichsweise hohen Wert. Die Parteiidentifikation hat daran aber keinen Anteil, da ihr Wert nicht signifikant ist. Dies ist insofern nicht überraschend, als das Die Linke 2009 zum ersten Mal im Saarland als Partei an einer Landtagswahl teilnahm. Zwar hatten die Wähler schon zur Bundestagswahl 2005 die Möglichkeit, sich für das Wahlbündnis aus WASG und PDS, aus dem Die Linke später hervorging, zu entscheiden; dass dies jedoch zu einer starken Bindung an die neue Partei führte, ist aufgrund des geringen Zeitraums von nur vier Jahren unwahrscheinlich. Die geringere Parteiidentifikation scheint somit logisch. Die Linke war im Saarland einfach noch nicht lange genug in Erscheinung getreten, um eine stabile Wählerbasis aufgebaut zu haben. Das heißt aber nicht, dass die Parteiidentifikation bei ihren Wählern gar nicht vorhanden ist. ${ }^{24}$

Auch wenn Die Linke zu einem nicht unwesentlichen Teil aus der PDS hervorgegangen ist und einige diese als „Nachfolgepartei“ ansehen, kann die Wählerschaft der PDS nicht jener der Linken gleichgesetzt werden. Dies ist insbesondere für Westdeutschland der Fall. Die PDS gewann ihre Wähler bis zur Bundestagswahl 2002 hauptsächlich aus der Klientel der ehemaligen DDR-Eliten und anderer vermeintlicher „Vereinigungsverlierer“ ${ }^{25}$ Die schlechten Wahlergebnisse der PDS in den westdeutschen Bundesländern, wie auch im Saarland, wo sie 2004 nur 2,3 Prozent der Stimmen erreichte, lassen sich hiermit erklären. Mit der Bundestagswahl 2005 und dem Wahlbündnis aus PDS und WASG kam es zu einem Wandel der Wählerschaft. Es gelang dem Wahlbündnis und später der Linken vermehrt, die

24 Auch wenn beim logistischen Modell für die Parteiidentifikation kein Einfluss nachgewiesen werden konnte, gab dennoch ein gutes Drittel der Befragten an, dass sie sich mit der Linken identifizieren.

25 Vgl. Oskar Niedermayer, Die Wählerschaft der Linkspartei.PDS 2005: Sozialstruktureller Wandel bei gleich bleibender politischer Positionierung, in: ZParl, 37. Jg. (2006), H. 3, S. 523 - 538, S. $525 \mathrm{ff}$. 
„natürlichen Wähler“ einer Linkspartei anzusprechen. Dies sind vor allem Arbeitslose und Bürger mit niedrigen und mittleren Bildungsabschlüssen. ${ }^{26}$ Damit entwickelte sich Die Linke, überspitzt formuliert, „,von einer Elitenpartei in Richtung einer Unterschichtpartei“27.

In der Wahrnehmung vieler Wähler wird die Bundestagswahl 2005 als „Geburtsstunde“ der Linken betrachtet. Bis zur Landtagswahl im Saarland 2009 hatte sie somit gerade vier Jahre Zeit, bei ihren Wählern auf eine Parteiidentifikation hinzuarbeiten. Folglich sind niedrige Werte für die Parteiidentifikation zu erwarten. In der Tat zeigen sich deutliche Unterschiede zwischen der Linken und den anderen Parteien (vgl. Tabelle 2): Nur 37,5 Prozent der Wähler der Linken gaben an, auch tatsächlich über eine Identifikation mit dieser Partei zu verfügen.

Noch interessanter als die zu erwartende relative geringe Identifikation mit der gewählten Partei ist der Wert für die Identifikation mit einer anderen Partei. Mit 27,3 Prozent gab knapp ein Drittel der befragten Linken-Wähler an, sich mit einer anderen Partei als der Linken zu identifizieren. Dabei weisen die meisten dieser Personen eine Parteiidentifikation zur SPD auf. Aber auch die Grünen und die CDU wurden genannt. Es ist anzunehmen, dass das Verhalten dieser Wähler von einem gewissen Protestgedanken getragen wurde. ${ }^{28}$

\begin{tabular}{|l|c|c|c|c|c|}
\hline \multirow{2}{*}{ Tabelle 2: Parteiidentifikation (PI) bei der saarländischen Landtagswahl 2009 (in Prozent) } \\
\hline & CDU & SPD & FDP & Grüne & Linke \\
\cline { 2 - 6 } & 78,6 & 78,6 & 63,9 & 63,6 & 37,5 \\
\hline PI zur gewählten Partei & 11,7 & 15,5 & 25,0 & 33,3 & 35,2 \\
\hline Keine PI & 9,7 & 6,0 & 11,1 & 3,0 & 27,3 \\
\hline PI zu anderen Parteien & Quelle: Eigene Berechnung auf Basis des MLP. \\
\hline
\end{tabular}

Auch bei den anderen Parteien finden sich Personen, die angaben, sich einer anderen als der gewählten Partei zugehörig zu fühlen. In diesen Fällen kann Protestwahlverhalten ebenfalls nicht ausgeschlossen werden, ist aber als unwahrscheinlicher zu betrachten. Um Protest zum Ausdruck zu bringen, werden vorzugsweise Parteien gewählt, die am Rande des politischen Spektrums stehen und / oder als nicht (vollständig) etablierte Parteien wahrgenommen werden. ${ }^{29}$ In den Fällen, bei denen eine Partei gewählt wurde, die nicht der Parteiidentifikation entspricht, es sich aber nicht um Die Linke handelt, ist deshalb eher zu vermuten, dass die Personen aus taktischen Gründen nicht der von ihr bevorzugten Partei ihre Stimme gegeben haben. ${ }^{30}$

26 Vgl. Viola Neu, Analyse der Bundestagswahl 2005, Berlin 2006, S. 26 ff.

27 Harald Schoen / Jürgen W. Falter, Die Wähler der Linkspartei, in: APuZ, B. $51 / 52$ (2005), S. 33 - 40, S. 38.

28 Vgl. Manuela Kulick / Holger Onken, Die Wähler der Linkspartei in ihren Hochburgen: eine empirische Analyse zur niedersächsischen Landtagswahl 2008 in Oldenburg, Delmenhorst und Wilhelmshaven, in: ZParl, 39. Jg. (2008), H. 2, S. 299 - 312.

29 Vgl. Franz Urban Pappi, Die Republikaner im Parteiensystem der Bundesrepublik - Protesterscheinung oder politische Alternative, in: APuZ, B 21 (1990), S. 37 - 44, S. 38 f.; Richard Hilmer / Rita Müller-Hilmer, Die Bundestagswahl vom 18. September 2005: Votum für Wechsel in Kontinuität, in: ZParl, 37. Jg. (2006), H. 1, S. 183-218.

30 Vgl. Harald Schoen, Wechselwahl, in: Jürgen W. Falter / ders. (Hrsg.), a.a.O. (Fn. 15), S. 367 - 387. 
Weiterhin erlaubt die Stärke der Parteiidentifikation Aussagen über die Verbundenheit zwischen Wählerschaft und Partei. Zwischen 67 und 80 Prozent der Wähler mit einer Parteiidentifikation gaben an, sich sehr oder ziemlich stark mit der Partei zu identifizieren. Dabei ist der Mittelwert bei den SPD-Wählern am geringsten, was mit einer gewissen Unzufriedenheit bei Teilen der Wählerschaft erklärt werden kann, während der Mittelwert für die Anhänger der Linken am höchsten ist. Deren starke Parteiidentifikation lässt sich mit dem Ideologiegrad erklären. Die Linke versucht auf dreierlei Wegen Wähler zu gewinnen: (1) Eine radikale Ideologie, über die eng mit der Partei verbundene Personen angesprochen werden; (2) die Programmatik. Mit der geforderten „sozialen Gerechtigkeit“ erreicht Die Linke eine große Gruppe von Wählern, die ähnliche realpolitische Vorstellungen wie sie selbst hat; (3) Protest, wobei davon auszugehen ist, dass diese Wähler keine Parteiidentifikation zu der Linken aufweisen. Der Ideologisierungsgrad der anderen Parteien - mit Ausnahme der Grünen - ist niedriger, wodurch die Wähler zwar über eine Parteiidentifikation verfügen, diese aber weniger stark ausgeprägt ist als bei der Linken.

\subsection{Issues}

Im Gegensatz zu der Parteiidentifikation, die als langfristiger Faktor so gut wie unabhängig von der jeweiligen Wahl ist, kann der Einfluss der Issues durch den Wandel der politischen Themenlandschaft von Wahl zu Wahl stark variieren. Damit ein Thema Einfluss auf den Wahlausgang nehmen kann, muss es zunächst vom Wähler wahrgenommen werden. Misst er ihr ausreichende Bedeutung bei und kann eine Partei mit diesem Problem beziehungsweise dessen Lösung in Verbindung bringen, so sind die notwendigen Voraussetzungen dafür geschaffen, dass ein Thema wahlentscheidenden Einfluss haben kann. Die Wirkungen von Wahlkampf und Medienberichterstattung sind dabei nicht zu vernachlässigen, da sie ihrerseits die Wahrnehmung der Themen beeinflussen. Empirisch belegt werden kann die Themenorientierung anhand der den Parteien zugeschriebenen Problemlösungskompetenz sowie über einen Vergleich zwischen den eigenen und den antizipierten Positionen der Parteien. ${ }^{31}$

In dem berechneten Regressionsmodell hatten die Issues mit einem Wert von 14,355 einen deutlichen, wenn auch nur vergleichsweise geringen Einfluss auf die Wahlentscheidung der Linken. Für die anderen Parteien, mit Ausnahme der CDU, waren Themen nicht signifikant, und bei der CDU war ihr Einfluss nur sehr gering.

Bei einer einfachen Betrachtung zeigt sich erwartungsgemäß, dass die Wähler die Lösungskompetenz für das wichtigste Problem zumeist jener Partei zusprechen, der sie auch ihre Stimme gaben (siehe Tabelle 3). Dies trifft auf alle Parteien - mit Ausnahme der Grünen - zu. Jeder zweite Wähler der Linken ist der Ansicht, dass diese das ihm jeweils wichtigste Problem lösen kann. Dabei war es sicher von Vorteil für die Linke, dass mit der Wirtschafts- und Finanzkrise sowie der Arbeitslosigkeit Probleme dominierten, die in ihrer

31 Kunz und Thaidigsmann zeigten in diesem Zusammenhang jedoch, dass die Problemlösungskompetenz bei der Bundestagswahl 2002 durchweg die stärkere Erklärungskraft besaß. Volker Kunz I Isabell Thaidigsmann, Die Relevanz von Themenorientierung für das Wählerverhalten bei der Bundestagswahl 2002, in: Jürgen W. Falter / Oscar W. Gabriel / Bernhard Weßels (Hrsg.), a.a.O. (Fn. 2), S. $50-76$. 
Programmatik eine wichtige Rolle einnehmen und auch während des Wahlkampfes immer wieder Teil ihrer Kampagne waren.

Immerhin knapp elf Prozent der Linken-Wähler trauen die Lösung des wichtigsten Problems der SPD zu. Vermutlich fühlen sich diese durchaus noch der SPD verbunden und gaben eher aus Protest der Linken ihre Stimme (dies lassen auch die Analysen zur Parteiidentifikation vermuten).

\begin{tabular}{|l|r|r|r|r|r|}
\hline Tabelle 3: Lösungskompetenz für das wichtigste Problem (in Prozent) \\
\hline \multirow{2}{*}{ Lösungskompetenz } & \multicolumn{5}{|c|}{ Wahlentscheidung Landtagswahl Saarland } \\
\cline { 2 - 6 } & CDU & SPD & FPD & Grüne & Linke \\
\hline CDU & 66,7 & 8,0 & 10,7 & 15,4 & 5,4 \\
\hline SPD & 2,2 & 53,3 & & & 10,8 \\
\hline FDP & 6,7 & 2,7 & 50,0 & 3,8 & 2,7 \\
\hline Grüne & 1,1 & 4,0 & 3,6 & 23,1 & 1,4 \\
\hline Linke & & & & 3,8 & 47,3 \\
\hline alle Parteien gleich gut & 4,4 & 9,3 & 3,6 & 11,5 & 2,7 \\
\hline keine Partei & 18,9 & 22,7 & 32,1 & 42,3 & 29,7 \\
\hline Quelle: Eigene Berechnung auf Basis des MLP. \\
\hline
\end{tabular}

Auffällig ist der hohe Anteil der Wähler, nämlich 33,6 Prozent, die die Ansicht vertreten, dass keine Partei das wichtigste Problem lösen kann. Die Wähler der Grünen liegen mit 42,3 Prozent dabei etwas über dem Durchschnitt und die Wähler der CDU mit 18,9 Prozent am weitesten darunter. Interessant sind auch die Aussagen der Befragten, auf welcher Ebene (europäisch, national, Bundesländer) das wichtigste Problem gelöst werden müsste. Nur 30,1 Prozent gaben hier die Bundesländer an. Demgegenüber vertraten 61,6 Prozent der Befragten die Ansicht, dass das wichtigste Problem auf nationaler Ebene gelöst werden muss, 8,3 Prozent sahen die Lösungskompetenz auf europäischer Ebene. Folglich ist es nicht verwunderlich, dass der Erklärungsgehalt des Faktors „Issues“ gering ist, da die Codierung auf der Variable „Problemlösungskompetenz“ beruht und diese vom Großteil der Befragten nicht auf Landesebene gesehen wird.

\subsection{Kandidaten}

Den mit Abstand stärksten Einfluss auf die Entscheidung der Linken-Wähler hatte ihr Kandidat: Oskar Lafontaine (vgl. Tabelle 1). Während die Kandidaten der anderen vier Parteien keinen Einfluss auf die Wahlentscheidung für Die Linke ausübten, ließen sich die Wähler der CDU und SPD von Lafontaine in dem Sinne beeinflussen, dass sie eben gerade seinetwegen der CDU beziehungsweise SPD ihre Stimme gaben. Hieraus kann jedoch nicht zwangsläufig gefolgert werden, dass diese Wähler der Linken ihre Stimme gegeben hätten, wenn diese mit einem anderen Kandidaten angetreten wäre. Vielmehr ist es möglich, dass diese Wähler ursprünglich den Grünen oder der FDP ihre Stimme geben wollten, dies aber unterließen, um die beiden größeren Parteien und damit auch deren Kandidaten und Konkurrenten um das Ministeramt zu unterstützen.

Wie schon das Regressionsmodell gezeigt hat, beeinflusste Lafontaine die Wahlentscheidung stärker, als es die Spitzenkandidaten der anderen Parteien vermochten. Ein weiterer 
deutlicher Unterschied ließ sich zwischen den beiden größeren Parteien (CDU und SPD) und den beiden kleineren Parteien (FDP und Grüne) feststellen. Auch wenn Faktoren wie persönliches Image und eine ausgeklügelte Kandidatenstrategie immer mehr an Bedeutung gewinnen $^{32}$, hatten die Spitzenpolitiker der FDP und der Grünen eine kaum wahrnehmbare Wirkung auf das Verhalten ihrer Wähler. Im Gegensatz zu den anderen drei Parteien stellten diese aber keinen Kandidaten für das Amt des Ministerpräsidenten auf - was den geringen Einfluss erklären kann. Daher werden in die folgende Betrachtung nur Peter Müller (CDU), Heiko Maas (SPD) und Oskar Lafontaine (Die Linke) einbezogen.

Als Ministerpräsident wurde von allen Befragten Müller favorisiert. Mit gut fünf Prozent Vorsprung lag er vor seinen beiden Kontrahenten, die von je 26,2 Prozent der Wähler als Präferenz für das Amt angegeben wurden. Trotz ungewöhnlich hoher Auswahl zwischen drei Kandidaten waren knapp 16 Prozent der Saarländer der Ansicht, dass sich keiner der drei Politiker für das Amt des Ministerpräsidenten eigne. Die Anhänger der Union befürworteten zu 90 Prozent ihren Kandidaten. Eine ausgeprägte Präferenz hatten zwar auch die Anhänger der SPD und der Linken für ihren jeweiligen Bewerber für das höchste Amt, jedoch waren jeweils circa zehn Prozent der Ansicht, der Kandidat der jeweils anderen „linken" Partei sei der geeignetere.

Neben der Frage, welcher Politiker als Ministerpräsident präferiert werde, wurden die Saarländer um eine Einschätzung der drei zur Wahl stehenden Kandidaten gebeten. Lafontaine wurde dabei deutlich negativer wahrgenommen, als seine Konkurrenten von CDU und SPD. Aber auch Peter Müller wurde von einem Viertel der Saarländer negativ bewertet, während Heiko Maas nicht so stark polarisierte, was sich auch an dem Mittelwert von 0,04 zeigt. ${ }^{33}$ Weder sahen ihn die Saarländer besonders positiv noch sehr negativ. Die Mittelwerte von Lafontaine wie auch Müller liegen im negativen Bereich, wobei Lafontaine im Durchschnitt mit -1,31 negativer bewertet wurde als Müller mit nur -0,39. Allerdings darf aufgrund des Mittelwertes nicht angenommen werden, dass die Saarländer Maas als Ministerpräsidenten vor Lafontaine und Müller bevorzugten. Vielmehr scheinen die beiden Letztgenannten die Wähler stärker in zwei Lager zu trennen, während nur vergleichsweise wenige ihnen neutral gegenüberstehen. Nach dem Motto „auch negative Werbung ist Werbung“ wurden Müller und Lafontaine wenn auch nicht von allen positiv, so doch zumindest von allen wahrgenommen, während Maas bei weitem nicht so präsent war. Dabei wurde Lafontaine verstärkt negativ eingeschätzt, während Müller mehr ihm positiv gesinnte Wähler auf sich vereinen konnte.

Der Vergleich der Mittelwerte nach Wählergruppen zeigt zudem, dass der eigene Kandidat von den Wählern der jeweiligen Partei bei der CDU und der Linken sehr positiv wahrgenommen wurde. Einzig Maas erhält auch von den SPD-Wählern keine guten Bewertungen. Auch dies ist als weiteres Indiz für die starke Position Lafontaines als wahlentscheidender Faktor zu werten. Es fiel den ehemaligen SPD-Wählern damit auch auf dieser Ebene leicht, sich für den aus ihren Augen besseren Kandidaten und somit für eine andere Partei zu entscheiden.

32 Vgl. Dieter Roth / Andreas M. Wüst, Abwahl ohne Machtwechsel: Die Bundestagswahl 2005 im Lichte langfristiger Entwicklungen, in: Eckard Jesse / Roland Sturm (Hrsg.), Bilanz der Bundestagswahl 2005: Voraussetzungen, Ergebnisse, Folgen, Wiesbaden 2006, S. 43 - 70.

33 Die Politiker-Skalometer wurden im MLP elfstufig abgefragt, wobei -5 „halte überhaupt nichts von der Person" bedeutete und 5 ,halte sehr viel von der Person“. 


\section{Stärke der Einflussfaktoren in anderen Bundesländern}

Es bleibt also festzuhalten: Der starke Einfluss des Kandidaten, die schwächere, aber vorhandene Wirkung der Issues sowie jegliches Fehlen eines Einflusses der Parteiidentifikation, verbunden mit einer hohen Erklärungskraft, zeigen deutliche Unterschiede zwischen der Linken und den anderen Parteien.

Das gute Ergebnis für Die Linke scheint folglich dem Kandidaten Oskar Lafontaine geschuldet zu sein. Ob dem aber wirklich so ist, lässt sich nur im Vergleich mit anderen Wahlergebnissen und deren Einflussfaktoren der bestimmen. Schließlich wäre es auch möglich, dass sich die Wähler der Linken generell von anderen Faktoren in ihrer Wahlentscheidung beeinflussen lassen als die Wähler der etablierten Parteien.

Aufgrund der Datenlage ist ein solcher Vergleich leider nur für die Entscheidung der Wähler in Sachsen, Thüringen und Brandenburg möglich. Auch wenn dies nicht optimal ist, da die Linke in den ostdeutschen Bundesländern bereits vollständig im Parteiensystem verankert ist und teilweise bessere Wahlergebnisse erzielt als die SPD oder CDU ${ }^{34}$, erscheint ein Vergleich lohnenswert. Das Parteiensystem in Deutschland ist derzeit im Wandel begriffen. Seit den Landtagswahlen im Jahr 2005 und der Bundestagswahl 2009 scheint Die Linke im westdeutschen Parteiensystem anzukommen und ist regelmäßig in den Landesparlamenten vertreten. Auch Koalitionen werden de facto nicht mehr ausgeschlossen. Ein erster Schritt ist die Minderheitsregierung im größten Bundesland Nordrhein-Westfalen, wo sich Hannelore Kraft nur durch Kooperation (Enthaltung) der Linken zur Ministerpräsidentin wählen lassen konnte.

\begin{tabular}{|l|c|c|c|c|}
\hline \multicolumn{5}{|c|}{ Tabelle 4: Wablergebnisse der Landtagswablen 2009 im Vergleich (in Prozent) } \\
\hline & Saarland & Sachsen & Brandenburg & Thüringen \\
\hline CDU & 34,5 & 40,2 & 19,8 & 31,2 \\
\hline SPD & 24,5 & 10,4 & 33,0 & 18,5 \\
\hline FDP & 9,2 & 10,0 & 7,2 & 7,6 \\
\hline Grüne & 5,9 & 6,4 & 5,7 & 6,2 \\
\hline Linke & 21,3 & 20,6 & 27,2 & 27,4 \\
\hline Quelle: Landeswahlleiter Saarland, Sachsen, Brandenburg, Thüringen. Eigene Zusammenstellung. \\
\hline
\end{tabular}

34 So wird in der politikwissenschaftlichen Diskussion auch von zwei verschiedenen deutschen Parteiensystemen gesprochen: Das westdeutsche Parteiensystem mit zwei großen (CDU/CSU, SPD) und zwei kleinen Parteien (FDP, Grüne), sowie einer fünften kleineren Partei, die aber lange als koalitionsunfähig eingestuft wurde. In Ostdeutschland dagegen stellt sich die Parteienlandschaft anders da. Nach der ersten gesamtdeutschen Wahl 1990 konnte von zwei mittelgroßen Parteien (CDU, SPD) und drei kleineren Parteien (PDS, Grüne, FDP) gesprochen werden. Die Gewichte verschoben sich aber schon nach der Bundestagswahl 1994, als die PDS in Ostdeutschland knapp 20 Prozent erreichte und nun - nach den Wahlergebnissen bei Bundestagswahlen in Ostdeutschland - von drei mittelgroßen (CDU, SPD, PDS/Linke) und zwei kleineren Parteien (Grüne, FDP) gesprochen werden kann. Vgl. Eckard Jesse, Zwei Parteiensysteme? Parteien und Parteiensystem in den alten und neuen Ländern vor und nach der Bundestagswahl 2002, in: ders. (Hrsg.), Bilanz der Bundestagswahl 2002 - Voraussetzungen, Ergebnisse, Folgen, Opladen / München 2003, S. $15-36$, S. 17. 
Bei einem Vergleich der Landtagswahlergebnisse der vier zu untersuchenden Bundesländer zeigen sich durchaus Ähnlichkeiten. So erreichte Die Linke im Saarland sogar ein leicht besseres Ergebnis als in Sachsen. Die Wahlerfolge der beiden großen Parteien variieren. Dennoch erreichen CDU und SPD zusammen in den drei ostdeutschen Bundesländern um die 50 Prozent der Wählerstimmen, im Saarland mit knapp 60 Prozent etwas mehr. Die Grünen liegen überall um sechs, die FDP um acht Prozent.

\begin{tabular}{|l|c|c|c|c|c|c|c|c|}
\hline \multicolumn{1}{|l|}{ Tabelle 5: Einfluss der Faktoren auf die Wablentscheidung der Linken-Wähler } \\
\hline & $\begin{array}{c}\text { Nagel- } \\
\text { kerkes } \mathrm{R}^{2}\end{array}$ & PI* $^{*}$ & Issue $^{*}$ & $\begin{array}{c}\text { Kandidat } \\
\text { CDU }\end{array}$ & $\begin{array}{c}\text { Kandidat } \\
\text { SPD }\end{array}$ & $\begin{array}{c}\text { Kandidat } \\
\text { FDP }\end{array}$ & $\begin{array}{c}\text { Kandidat } \\
\text { Grüne }\end{array}$ & $\begin{array}{c}\text { Kandidat } \\
\text { Linke }\end{array}$ \\
\hline Saarland & 0,671 & 2,518 & $14,355^{\mathrm{b}}$ & 0,189 & 0,149 & 2,382 & 0,337 & $230,07^{\mathrm{a}}$ \\
\hline Sachsen & 0,711 & $278,406^{\mathrm{a}}$ & 5,435 & $0,033^{\mathrm{b}}$ & 5,491 & 0,067 & 3,241 & 4,686 \\
\hline Brandenburg & 0,544 & $25,841^{\mathrm{a}}$ & $14,323^{\mathrm{b}}$ & $0,004^{\mathrm{c}}$ & $22,493^{\mathrm{b}}$ & 1,702 & 0,993 & $44,087^{\mathrm{c}}$ \\
\hline Thüringen & 0,664 & $571,596^{\mathrm{a}}$ & $8,347^{\mathrm{b}}$ & $0,182^{\mathrm{c}}$ & $0,026^{\mathrm{b}}$ & 0,044 & $29,723^{\mathrm{c}}$ & $53,090^{\mathrm{b}}$ \\
\hline $\begin{array}{l}\text { * Parteiidentifikation / Issueorientierung zur gewählten Partei. } \\
\text { Signifikanzen: a: } \mathrm{p}<0,000 ; \mathrm{b}: \mathrm{p}<0,01 \text {; c: } \mathrm{p}<0,05 .\end{array}$ \\
Quelle: Eigene Berechnung auf Basis des MLP. \\
\hline
\end{tabular}

Die Betrachtung der Einflussfaktoren auf die Wahlentscheidung für Die Linke zeigt im Gegensatz dazu deutliche Unterschiede zwischen den ostdeutschen Bundesländern und dem westdeutschen Saarland. In Sachsen und Thüringen dominiert der Einfluss der Parteiidentifikation, der für das Saarland gar nicht nachgewiesen werden konnte. Die Linke hat in den ostdeutschen Bundesländern (als PDS) eine längere Tradition, so dass sie viel stärkere Bindungen zu ihren Wählern aufbauen konnte.

In Thüringen zeigt sich darüber hinaus ein Einfluss des eigenen Kandidaten Bodo Ramelow und der Kandidatin der Grünen, Astrid Rothe-Beinlich (im Vergleich zur Parteiidentifikation jedoch relativ gering, was auch mit dem nur geringen Bekanntheitsgrad der Kandidatin erklärt werden kann). Ihre Wirkung muss dabei im Hinblick auf eine potentielle Koalitionsbildung betrachtet werden. Schließlich kam eine Koalition aus CDU und FDP, eine Große Koalition oder eine rot-rot-grüne Koalition in Betracht, die von den drei möglichen beteiligten Parteien vor der Wahl nicht ausgeschlossen worden war. Wie im Saarland haben zudem auch in Thüringen die Themen Einfluss auf das Wahlverhalten gehabt, allerdings in ungleich geringem Maße.

Einige Parallelen zum Saarland lassen sich auch in Brandenburg finden. In beiden Bundesländern ging die stärkste Wirkung von dem Kandidaten der Linken aus - in Brandenburg Kerstin Kaiser, im Saarland Oskar Lafontaine. Ansonsten unterscheiden sich die Einflussfaktoren aber erheblich. Beispielsweise hatte in Brandenburg der SPD-Kandidat (Matthias Platzeck) großen Einfluss auf das Wahlverhalten, was sich mit seiner generellen Beliebtheit im Land erklären lässt. Zudem war er einer Zusammenarbeit mit der Linken nicht abgeneigt, die nach der Wahl auch umgesetzt wurde. Sachthemen beeinflussten in Brandenburg wie auch im Saarland die Wahlentscheidung. Allerdings war dies der Faktor mit dem geringsten Einfluss, was sich sicherlich auch durch die föderale Struktur der Bundesrepublik erklären lässt.

Bei einem Vergleich der Faktoren für die Wahlentscheidung zwischen den Wählern der Linken in verschiedenen Bundesländern können also Differenzen zwischen dem Saarland 
und dem Rest festgestellt werden. In den Vergleichsbundesländern ist die Parteiidentifikation von größerer Bedeutung für die Wahlentscheidung, die Issues dagegen üben nur geringen Einfluss aus. Die Kandidaten beeinflussen in allen Bundesländern die Wahlentscheidung, aber nur in einem Fall ist dieser Faktor gewichtiger als die Parteiidentifikation. Die Erklärungskraft aller untersuchten Faktoren zusammen ist auf ähnlich hoher Ebene vorhanden. Im Gegensatz zu diesem Befund wurde die Wahlentscheidung der Saarländer für Die Linke vom Einfluss des Kandidaten dominiert. Themen machten nur einen geringen Anteil aus.

\section{Der Sonderfall ist möglich: Kandidat wichtiger als Parteiidentifikation}

Das Wahlverhalten der Linken-Anhänger bei der saarländischen Landtagswahl 2009 unterschied sich deutlich von dem der Wähler der anderen Parteien. War der Linken im Saarland bisher nicht genügend Zeit geblieben, um eine stabile Wählerbasis aufzubauen, so konnte sie dieses Defizit durch den sehr starken Bewerber für das Amt des Ministerpräsidenten wieder gut machen. Der ehemalige SPD-Ministerpräsident konnte als Kandidat der Linken die Menschen überzeugen. Wo Heiko Maas versagte, triumphierte Oskar Lafontaine. Der ehemalige SPD-Vorsitzende und Ministerpräsident des Saarlandes schaffte es nicht nur, die Anhänger seiner eigenen Partei zu begeistern, sondern konnte auch viele ehemalige SPDWähler für sich gewinnen. Zudem wurden viele Nichtwähler wieder an die Wahlurnen gelockt.

So stark seine Anhänger sich für Lafontaine begeistern ließen, so unsympathisch fanden ihn die Wähler der anderen Parteien. Trotz dieser Polarisierung hatte Die Linke offensichtlich den richtigen Kandidaten für die Landtagswahl aufgestellt. Ohne ihn als Zugpferd wäre es für die Partei sicher schwer gewesen, ein ähnliches Ergebnis zu erzielen. Gepaart mit einer günstigen Wahrnehmung des wichtigsten Problems, bei dem gehäuft die Kernthemen der Linken genannt wurden, ergab sich so bei dieser Wahl eine für sie günstige Konstellation. Am Ende erreichte sie ihr bislang bestes Wahlergebnis in Westdeutschland. Der „Verräter" wurde vom Wahlvolk geliebt.

Über künftige Wahlen lässt sich aus diesem Ergebnis heraus jedoch keine Prognose formulieren. Der Kandidat Lafontaine hatte bei dieser Wahl solch einen großen Einfluss auf das Ergebnis, dass unklar bleibt, ob die einmalige Mobilisierung durch ihn schon ausreichend war, um die Wähler dauerhaft an Die Linke zu binden. Auch zeigt sich im Vergleich der Einflussfaktoren in anderen Bundesländern, dass der Kandidat im Normalfall nicht einen derart hohen Einfluss auf die Wahlentscheidung ausübt, sondern diese vielmehr - wie auch bei den anderen Parteien - stark durch die Parteiidentifikation geprägt wird. Wie gesehen ist es jedoch möglich, dass die Wahl einer Partei maßgeblich vom Spitzenkandidaten abhängt und dieser Faktor unter Umständen sogar entscheidender ist als die „normalerweise" alles überlagernde Parteiidentifikation. 